\title{
LIFTING TO MAXIMAL RIGID OBJECTS IN 2-CALABI-YAU TRIANGULATED CATEGORIES
}

\author{
YUNLI XIE AND PIN LIU \\ (Communicated by Birge Huisgen-Zimmermann)
}

\begin{abstract}
We show that a tilting module over the endomorphism algebra of a maximal rigid object in a 2-Calabi-Yau triangulated category lifts to a maximal rigid object in this 2-Calabi-Yau triangulated category. This strengthens recent work of $\mathrm{Fu}$ and Liu for cluster-tilting objects.
\end{abstract}

\section{INTRODUCTION}

Tilting theory in cluster categories and, more generally, Hom-finite Calabi-Yau triangulated categories have recently been widely investigated. The study of such categories was originally motivated by their links to cluster algebras, and indeed there has been a considerable amount of activity and a lot of results in this direction. We refer the reader to the recent survey $[\mathrm{K}]$.

However, the study of such categories has also contributed to new developments in the theory of finite-dimensional (and, more generally, non-commutative) algebras. The endomorphism algebras of cluster-tilting objects in the Calabi-Yau triangulated categories have been further developed, and it has been revealed that they have very nice properties (see $[\mathrm{KR}$ for instance). Cluster-tilting objects are always maximal rigid objects, while the converse is not true in general. There exist 2Calabi-Yau triangulated categories in which maximal rigid objects are not cluster tilting. The first examples of such categories were given by Burban-Iyama-KellerReiten in BIKR. Cluster tubes, introduced by Barot-Kussin-Lenzing in BKL, are another family of 2-Calabi-Yau triangulated categories without cluster-tilting objects. In [BMV] Buan-Marsh-Vatne classified maximal rigid objects of cluster tubes, none of which is cluster tilting. The endomorphism algebras of maximal rigid objects in the 2-Calabi-Yau triangulated categories are studied in $\mathrm{V}, \mathrm{Y}, \mathrm{ZZ}$.

It is an interesting idea to investigate algebras derived equivalent to the endomorphism algebras of cluster-tilting objects and maximal rigid objects. Understanding their tilting modules is a step in this direction ([FL, [HJ], $\mathrm{S}]$ ). The aim of this note is to give an answer as to how to identify tilting modules over the endomorphism algebras of maximal rigid objects in 2-Calabi-Yau triangulated categories.

Received by the editors May 10, 2011 and, in revised form, December 13, 2011.

2010 Mathematics Subject Classification. Primary 18E30, 16D90.

Key words and phrases. 2-Calabi-Yau category, tilting modules, maximal rigid objects.

The first author was supported by the NSF of China (Grant 11026190) and the Fundamental Research Funds for the Central Universities (Grants SWJTU11BR098, SWJTU12CX056, and SWJTU12ZT15).

The second author is the corresponding author. 
We show that tilting modules over such algebras lift to maximal rigid objects in the corresponding 2-Calabi-Yau triangulated category. Namely, we prove the following theorem.

Theorem. Let $R$ be a maximal rigid object in the 2-Calabi-Yau triangulated category $\mathcal{C}, \Gamma$ be the endomorphism algebra of $R$ and $T$ be a tilting module over $\Gamma$. Then $T$ lifts to a maximal rigid object in $\mathcal{C}$.

\section{Preliminaries}

In this section we review some useful notation and results.

1.1. Tilting modules. Let $k$ be an algebraically closed field and $A$ be a finitedimensional algebra. Let $\bmod A$ be the category of finite-dimensional right $A$ modules. For an $A$-module $T$, let add $T$ denote the full subcategory of $\bmod A$ with objects all direct summands of direct sums of copies of $T$. Then $T$ is called a tilting module in $\bmod A$ if

$-\operatorname{pd}_{A} T \leq 1$,

- $\operatorname{Ext}_{A}^{1}(T, T)=0$,

- there is an exact sequence $0 \rightarrow A \rightarrow T^{0} \rightarrow T^{1} \rightarrow 0$, with $T^{0}, T^{1}$ in add $T$.

This is the original definition of tilting modules from [HR], and it was proved in B] that the third axiom can be replaced by the following:

- the number of indecomposable direct summands of $T$ (up to isomorphism) is the same as the number of simple $A$-modules.

1.2. Approximation. Let $\mathcal{A}$ be an additive category and $\mathcal{B}$ be an additive subcategory of $\mathcal{A}$. For an object $A$ in $\mathcal{A}$, a map $f: B \rightarrow A$ with $B \in \mathcal{B}$ is called a right $\mathcal{B}$-approximation if for all objects $B^{\prime}$ in $\mathcal{B}$, the sequence $\operatorname{Hom}_{\mathcal{C}}\left(B^{\prime}, B\right) \rightarrow$ $\operatorname{Hom}_{\mathcal{C}}\left(B^{\prime}, A\right) \rightarrow 0$ is exact. A minimal right $\mathcal{B}$-approximation is a right $\mathcal{B}$-approximation $f: B \rightarrow A$ such that for every $g: B \rightarrow B$ such that $f g=f$, the map $g$ is an isomorphism. Dually, we have the concepts of left $\mathcal{B}$-approximations and minimal left $\mathcal{B}$-approximations.

The full subcategory $\mathcal{B}$ is called functorially finite if for every object $A$ in $\mathcal{A}$, there exist a right $\mathcal{B}$-approximation ending in $A$ and a left $\mathcal{B}$-approximation starting in $A$. This notation was introduced in $[\mathrm{AS}$.

The following is well-known and straightforward to check.

Lemma 1.1. Let $\mathcal{B}$ be a full additive subcategory of $\mathcal{A}$ and $A$ an object of $\mathcal{A}$. If there is a right $\mathcal{B}$-approximation of $A$, then there is a minimal right $\mathcal{B}$-approximation of $A$, unique up to isomorphism.

1.3. Maximal rigid objects in 2-Calabi-Yau triangulated categories. Let $k$ be an algebraically closed field and $\mathcal{C}$ be a Krull-Schmidt triangulated $k$-linear category with split idempotents and suspension functor $S$. We suppose that all Hom-spaces of $\mathcal{C}$ are finite-dimensional and that $\mathcal{C}$ admits a Serre functor $\Sigma$; i.e., for any $X, Y$ in $\mathcal{C}$, we have the following bifunctorial isomorphisms:

$$
\operatorname{Hom}_{\mathcal{C}}(X, Y) \simeq D \operatorname{Hom}_{\mathcal{C}}(Y, \Sigma X)
$$

where $D=\operatorname{Hom}_{k}(-, k)$ is the usual duality. We suppose that $\mathcal{C}$ is Calabi-Yau of $\mathrm{CY}$-dimension 2; i.e. there is an isomorphism of triangle functors

$$
S^{2} \stackrel{\sim}{\rightarrow} \Sigma \text {. }
$$


For $X, Y \in \mathcal{C}$ and $n \in \mathbb{Z}$, we put as usual

$$
\operatorname{Ext}_{\mathcal{C}}^{n}(X, Y)=\operatorname{Hom}_{\mathcal{C}}\left(X, S^{n} Y\right) .
$$

Thus the Calabi-Yau property can be written as the following bifunctorial isomorphisms:

$$
D \operatorname{Ext}^{1}(X, Y) \simeq \operatorname{Ext}^{1}(Y, X) \text {, for any } X, Y \text {. }
$$

An object $R$ of $\mathcal{C}$ is rigid if $\operatorname{Ext}_{\mathcal{C}}^{1}(R, R)=0$. It is maximal rigid if it is rigid and $\operatorname{Ext}_{\mathcal{C}}^{1}(X \oplus R, X \oplus R)=0$ implies that $X \in$ add $R$, where add $R$ denotes the additive closure of $R$. An object $M$ of $\mathcal{C}$ is finitely presented by $R$ if there is a triangle in $\mathcal{C}$

$$
R_{1} \rightarrow R_{0} \stackrel{f}{\rightarrow} M \rightarrow S R_{1}
$$

with $R_{0}, R_{1}$ in add $R$. The morphism $f$ is necessarily a right add $R$-approximation of $M$, and, conversely, the cone of any add $R$-approximation of an object $M$ finitely presented by $R$ belongs to add $S R$ (see $[\mathrm{Y}]$ ). Let $\operatorname{pr}(R)$ denote the (full) subcategory of $\mathcal{C}$ of objects finitely presented by $R$.

Let $\Gamma$ be the endomorphism algebra of $R$. Let $\bmod \Gamma$ denote the category of finite-dimensional right modules over $\Gamma$. The following result describes the relationship between a 2-CY triangulated category and the corresponding endomorphism algebra of a maximal rigid object (see $[\mathrm{Y}]$ ).

Lemma 1.2. The functor $F=\operatorname{Hom}_{\mathcal{C}}(R,-): \mathcal{C} \rightarrow \bmod \Gamma$ induces an equivalence

$$
\operatorname{pr}(R) / \operatorname{add} S R \stackrel{\sim}{\rightarrow} \bmod \Gamma,
$$

where the category on the left has the same objects as $\operatorname{pr}(R)$, with morphisms given by morphisms in $\mathcal{C}$ modulo maps factoring through add $S R$.

Thus the functor $F$ induces an equivalence from add $R$ to the category of projective modules in $\bmod \Gamma$.

Moreover, it is shown in [ZZ that all maximal rigid objects have the same number of pairwise non-isomorphic indecomposable direct summands. That is,

Lemma 1.3. All maximal rigid objects in the 2-Calabi-Yau triangulated category have the same number of indecomposable direct summands (up to isomorphism).

\section{MAIN RESUlT}

As before, let $R$ be a maximal rigid object in the 2-Calabi-Yau triangulated category $\mathcal{C}$, let $\Gamma$ be the endomorphism algebra of $R$ in $\mathcal{C}$ and let $F$ be the functor $\operatorname{Hom}_{\mathcal{C}}(R,-): \mathcal{C} \rightarrow \bmod \Gamma$. Notice that $F$ is dense, so for each $\Gamma$-module $M^{+}$there is some object $M \in \operatorname{pr}(R)$ with $F M=M^{+}$. We write $\mathcal{C}(X, Y)$ for the set of morphisms from $X$ to $Y$ in the category $\mathcal{C}$. In this section, we prove that the tilting modules over $\Gamma$ lift to maximal rigid objects in $\mathcal{C}$.

We need the following crucial proposition.

Proposition 2.1. Let $M^{+}, N^{+}$be two $\Gamma$-modules of projective dimension at most one and let $M, N$ be two objects in $\mathcal{C}$ corresponding to $M^{+}, N^{+}$respectively. If

$$
\operatorname{Ext}_{\Gamma}^{1}\left(M^{+}, N^{+}\right)=0 \text { and } \operatorname{Ext}_{\Gamma}^{1}\left(N^{+}, M^{+}\right)=0,
$$

then $\operatorname{Ext}_{\mathcal{C}}^{1}(M, N)=0$ and $\operatorname{Ext}_{\mathcal{C}}^{1}(N, M)=0$. 
Proof. Let $\tilde{S}(N, S M)$ be the class of maps $\phi: N \rightarrow S M$ which factor through an object from add $S R$.

First we claim that $\tilde{S}(N, S M)=0$. In fact, combining this with Lemma 1.1, we have the following "minimal add $R$-approximation" triangle:

$$
R_{1}^{N} \stackrel{g}{\rightarrow} R_{0}^{N} \stackrel{f}{\rightarrow} N \rightarrow S R_{1}^{N},
$$

where $f$ is the minimal right add $R$-approximation of $N$. Since $R$ is rigid, it is not hard to check that $\tilde{S}(N, S M)$ coincides with the kernel of

$$
\mathcal{C}(N, S M) \stackrel{\mathcal{C}(f, S M)}{\longrightarrow} \mathcal{C}\left(R_{0}^{N}, S M\right) .
$$

Applying the functor $\operatorname{Hom}_{\mathcal{C}}(-, M)$ to the triangle

$$
S^{-} R_{0}^{N} \stackrel{-S^{-} f}{\longrightarrow} S^{-} N \rightarrow R_{1}^{N} \stackrel{g}{\rightarrow} R_{0}^{N},
$$

one gets the following exact sequence:

$$
\mathcal{C}\left(R_{0}^{N}, M\right) \stackrel{\mathcal{C}(g, M)}{\longrightarrow} \mathcal{C}\left(R_{1}^{N}, M\right) \rightarrow \mathcal{C}\left(S^{-} N, M\right) \stackrel{\mathcal{C}\left(S^{-} f, M\right)}{\longrightarrow} \mathcal{C}\left(S^{-} R_{0}^{N}, M\right) .
$$

Thus we have

$$
\tilde{S}(N, S M)=\operatorname{Ker} \mathcal{C}(f, S M) \simeq \operatorname{Ker} \mathcal{C}\left(S^{-} f, M\right) \simeq \operatorname{Coker} \mathcal{C}(g, M) .
$$

Applying the functor $F$ to the triangle

$$
R_{1}^{N} \stackrel{g}{\rightarrow} R_{0}^{N} \stackrel{f}{\rightarrow} N \rightarrow S R_{1}^{N},
$$

we get the following projective resolution of $N^{+}$in $\bmod \Gamma$ :

$$
0 \rightarrow F R_{1}^{N} \stackrel{F g}{\rightarrow} F R_{0}^{N} \rightarrow N^{+} \rightarrow 0,
$$

since we consider the minimal add $R$-approximation triangle and $N^{+}$is of projective dimension at most 1 . Thus

$$
\operatorname{Ext}_{\Gamma}^{1}\left(N^{+}, M^{+}\right)=\text {Coker } \operatorname{Hom}_{\Gamma}\left(F g, M^{+}\right) .
$$

Note that $R$ is rigid, which implies that

$$
\text { Coker } \operatorname{Hom}_{\Gamma}\left(F g, M^{+}\right)=\operatorname{Coker} \mathcal{C}(g, M)
$$

by the definition of quotient category. Combining this with $(*)$, one gets

$$
\tilde{S}(N, S M) \simeq \operatorname{Ext}_{\Gamma}^{1}\left(N^{+}, M^{+}\right)=0 .
$$

Alternatively, if we denote by $\tilde{S}(M, S N)$ the class of maps $\phi: M \rightarrow S N$ which factor through an object from add $S R$, then $\tilde{S}(M, S N)=0$.

As before, we have the following minimal add $R$-approximation triangle for $M$ :

$$
R_{1}^{M} \rightarrow R_{0}^{M} \rightarrow M \stackrel{\beta}{\rightarrow} S R_{1}^{M} .
$$

Applying the functor $\operatorname{Hom}_{\mathcal{C}}(N,-)$ to the triangle

$$
S R_{1}^{M} \rightarrow S R_{0}^{M} \stackrel{\alpha}{\rightarrow} S M \rightarrow S^{2} R_{1}^{M}
$$

we get the exact sequence

$$
\mathcal{C}\left(N, S R_{0}^{M}\right) \stackrel{\mathcal{C}(N, \alpha)}{\longrightarrow} \mathcal{C}(N, S M) \rightarrow \mathcal{C}\left(N, S^{2} R_{1}^{M}\right) .
$$

Since

$$
\operatorname{Im} \mathcal{C}(N, \alpha) \subset \tilde{S}(N, S M)=0
$$


we get the injective map

$$
\mathcal{C}(N, S M) \hookrightarrow \mathcal{C}\left(N, S^{2} R_{1}^{M}\right),
$$

which can be written as follows by the definition of Serre functor and the 2-CalabiYau property:

$$
D \mathcal{C}(M, S N) \hookrightarrow D \mathcal{C}\left(S R_{1}^{M}, S N\right) .
$$

Thus the dual $\mathcal{C}\left(S R_{1}^{M}, S N\right) \rightarrow \mathcal{C}(M, S N)$ of the injective map is surjective, which means that

$$
\mathcal{C}(M, S N) \simeq \operatorname{Im} \mathcal{C}(\beta, S N) \subset \tilde{S}(M, S N) .
$$

That is, $\operatorname{Ext}_{\mathcal{C}}^{1}(M, N)=0$. Thanks to the 2-Calabi-Yau property, we get $\operatorname{Ext}_{\mathcal{C}}^{1}(N, M)$ $=0$, too.

By the definition of quotient category, we can use the same notation for a $\Gamma$ module and its preimage in $\mathcal{C}$ under the projection $\operatorname{pr}(R) \rightarrow \operatorname{pr}(R) /$ add $S R \stackrel{\sim}{\rightarrow}$ $\bmod \Gamma$. We now prove our main result.

Theorem 2.2. Let $R$ be a maximal rigid object in the 2-Calabi-Yau triangulated category $\mathcal{C}$ and let $\Gamma$ be the endomorphism algebra of $R$. Let $T$ be a tilting module over $\Gamma$; then $T$ lifts to a maximal rigid object in $\mathcal{C}$.

Proof. Note that $T$ is a tilting module over $\Gamma$, so $T$ has no self-extension in $\mathcal{C}$ by Proposition 2.1. That is, $T$ is rigid in $\mathcal{C}$.

Suppose that $T$ is not maximal, which means that there exists $X \operatorname{not}$ from $\operatorname{add} T$ such that $\operatorname{Ext}_{\mathcal{C}}^{1}(X \oplus T, X \oplus T)=0$. On the other hand, because $T$ is a tilting module, the number of indecomposable direct summands of $T$ (up to isomorphism) is the same as the number of simple $\Gamma$-modules which is the number of indecomposable direct summands of $R$. Thus the number of pairwise non-isomorphic indecomposable direct summands of the rigid object $X \oplus T$ is greater than that of the maximal rigid object $R$, which is a contradiction to Lemma 1.3 .

\section{The Cluster-tilting obJects CASE}

Cluster-tilting objects in 2-Calabi-Yau triangulated categories and the corresponding endomorphism algebras were originally defined and studied in $[\mathrm{KR}$. Let $\mathcal{C}$ be a 2-Calabi-Yau triangulated category. An object $T$ in $\mathcal{C}$ is called cluster tilting if it satisfies that

- $\operatorname{Ext}_{\mathcal{C}}^{1}(T, T)=0$ and

- for any $X \in \mathcal{C}$, if $\operatorname{Hom}_{\mathcal{C}}(X, S T)=0$, then $X$ belongs to add $T$.

Any 2-Calabi-Yau triangulated category admits rigid objects (0 is viewed as a trivial rigid object) and also admits maximal rigid objects if $\mathcal{C}$ is skeletally small. As described in the introduction, there are 2-Calabi-Yau triangulated categories which contain no cluster-tilting objects. Cluster-tilting objects are obviously maximal rigid objects. But the converse is not true in general (see more in BIKR, BMV, KZ] ). Zhou-Zhu [ZZ proved that the converse is true if the 2-CalabiYau triangulated category admits a cluster-tilting object.

Lemma 3.1 (Zhou-Zhu). If the 2-Calabi-Yau triangulated category admits a cluster-tilting object, then every maximal rigid object is cluster tilting. 
Thus if the 2-Calabi-Yau triangulated category $\mathcal{C}$ admits a cluster-tilting object, our result indicates that the tilting modules over the endomorphism algebra of a cluster-tilting object in $\mathcal{C}$ lift to cluster-tilting objects in this 2-Calabi-Yau triangulated category. This coincides with Fu-Liu's result in $\mathrm{FL}$.

Theorem 3.2. Let $\mathcal{C}$ be a 2-Calabi-Yau triangulated category with a cluster-tilting object $T$ and let $\Gamma$ be the endomorphism algebra of $T$. Let $L$ be a tilting module over $\Gamma$; then $L$ lifts to a cluster-tilting object in $\mathcal{C}$.

Proof. This follows directly from Theorem 2.2 in combination with Lemma 3.1 .

Notice that Fu-Liu's proof is heavily based on the fact that the Auslander-Reiten translation of the 2-CY-tilted algebra (the endomorphism algebra of a cluster-tilting object in the 2-Calabi-Yau triangulated category) is induced by the AuslanderReiten translation of the 2-Calabi-Yau triangulated category. But this is not true for the endomorphism algebra of a maximal rigid object, since $\tau_{\mathcal{C}}$, the Auslander-Reiten translation of 2-Calabi-Yau triangulated category, is not closed in the subcategory $\operatorname{pr}(R)$ generally. The techniques used in this note are totally different from Fu-Liu's work, and our result strengthens theirs.

\section{ACKNOWLEDGMENTS}

Both of the authors learned cluster theory from Idun Reiten during their stay at NTNU. They are very grateful to Professor Idun Reiten for her guidance and constant support. They thank the algebra group at NTNU for their great hospitality. This work was inspired by some discussions with Changjian Fu. The authors thank him very much.

\section{REFERENCES}

[AS] Auslander, M., Smalø, S. O. (1980). Preprojective modules over Artin algebras. J. Algebra 66(1): 61-122. MR591246(83a:16039)

[B] Bongartz, K. (1981). Tilted algebras. Representations of algebras (Puebla, 1980), Lecture Notes in Math. 903. Berlin-New York: Springer, pp. 26-38. MR654701 (83g:16053)

[BIKR] Burban, I., Iyama, O., Keller, B., Reiten, I. (2008). Cluster tilting for one-dimensional hypersurface singularities. Adv. Math. 217(6): 2443-2484. MR2397457 (2010d:16012)

[BKL] Barot, M., Kussin, D., Lenzing, H. (2008). The Grothendieck group of a cluster category. J. Pure Appl. Algebra 212(1): 33-46. MR2355032 (2008j:18010)

[BMV] Buan, A. B., Marsh, R. J., Vatne, D. F. (2010). Cluster structure from 2-Calabi-Yau categories with loops. Math. Z. 265(4): 951-970. MR2652543(2011h:18014)

[FL] Fu, C., Liu, P. (2009). Lifting to cluster-tilting objects in 2-Calabi-Yau triangulated categories. Comm. Algebra 37: 1-9. MR2536929 (2010k:18015)

[HJ] Holm, T., Jorgensen, P. (2010). On the relation between cluster and classical tilting. J. Pure Appl. Algebra 214(9): 1523-1533. MR2593680 (2012b:16039)

[HR] Happel, D., Ringel, C. M. (1982). Tilted algebras. Trans. Amer. Math. Soc. 274(2): 399443. MR675063 (84d:16027)

[K] Keller, B. (2010). Cluster algebras, quiver representations and triangulated categories. London Math. Soc. Lecture Note Ser., 375, Cambridge Univ. Press. MR2681708 (2011h:13033)

[KR] Keller, B., Reiten, I. (2007). Cluster-tilted algebras are Gorenstein and stably Calabi-Yau. Adv. Math. 211(1): 123-151. MR2313531 (2008b:18018)

[KZ] Koenig, S., Zhu, B. (2008). From triangulated categories to abelian categories: cluster tilting in a general framework. Math. Z. 258: 143-160. MR2350040 (2008m:18021) 
[S] Smith, D. (2008). On tilting modules over cluster-tilted algebras. Illinois J. Math. 52(4): 1223-1247. MR2595764 (2011c:16042)

[V] Vatne, D. F. (2011). Endomorphism rings of maximal rigid objects in cluster tubes. Colloq. Math. 123: 63-93. MR2794120

[Y] Yang, D. (2012). Endomorphism algebras of maximal rigid objects in cluster tubes. Comm. Algebra 40: 4347-4371. MR2989650

[ZZ] Zhou, Y., Zhu, B. (2011). Maximal rigid subcategories in 2-Calabi-Yau triangulated categories. J. Algebra 348: 49-60. MR2852231 (2012j:16034)

Department of Mathematics, Sichuan University, 610064 Chengdu, People's Republic of China-And-Department of Mathematics, Southwest Jiaotong University, 610031 Chengdu, People's Republic of China

E-mail address: xieyunli@home.swjtu.edu.cn

Department of Mathematics, Southwest Jiaotong University, 610031 Chengdu, PeoPLE'S REPUBlic OF CHINA

E-mail address: liupin@home.swjtu.edu.cn 